\title{
Pensar sobre la Morfología de las Palabras: un Proyecto Didáctico para el Desarrollo de Vocabulario en la Escuela Secundaria
}

\section{Thinking about the Morphology of Words: A Didactic Project to Enhance Vocabulary Development in Middle School}

\author{
Karina Hess Zimmermann * \\ Universidad Autónoma de Querétaro, México
}

\begin{abstract}
Diversos estudios muestran que existe una estrecha relación entre el nivel de vocabulario y el desarrollo conceptual, la presencia de habilidades lingüísticas complejas y la comprensión lectora en los individuos. No obstante, se sabe que no todos los estudiantes ingresan a la escuela con el mismo bagaje léxico, lo que puede representar una desventaja académica para algunos en relación con sus pares. Como respuesta a la problemática anterior, este artículo presenta el diseño, implementación y evaluación de un proyecto didáctico sobre la enseñanza de estrategias de análisis morfológico de palabras para incrementar las habilidades para aprender vocabulario en estudiantes de tercer grado de secundaria. Para ello se contó con un grupo experimental (19 alumnos) que participó en el proyecto didáctico focal y un grupo control (19 alumnos) que realizó otro proyecto de lengua. Para evaluar la efectividad del proyecto didáctico, ambos grupos se enfrentaron a un pre y pos-test sobre análisis morfológico de palabras. Los resultados muestran un incremento estadísticamente significativo en el grupo experimental y no en el grupo control tanto en la capacidad para segmentar palabras en sus morfemas como en la habilidad para extraer el significado de palabras y morfemas.
\end{abstract}

Palabras clave: Desarrollo de vocabulario; Enseñanza de la lengua materna; Proyecto educativo; Escuela secundaria; Morfología.

\begin{abstract}
Numerous studies show a close relationship between vocabulary and conceptual development, complex linguistic skills and reading comprehension in individuals. However, it is known that not all students start school with the same lexical knowledge, which can be an academic disadvantage for some in relation to their peers. As a response to the aforementioned problem, this article presents the design, implementation and evaluation of a didactic project aiming to increase vocabulary learning skills in adolescents in third grade of secondary school through the teaching of morphological word analysis strategies. An experimental group (19 students) participated in the focal didactic project and a control group (19 students) took part in another language project. To evaluate the effectiveness of the didactic project, both groups completed a pre-test and a post-test on morphological word analysis. The results of the experimental group show a statistically significant increase both in the ability to segment words in their morphemes and in the ability to extract the meaning of words and morphemes, while the results for the control group do not show significant differences.
\end{abstract}

Keywords: Vocabulary development; Mother tongue instruction; Educational projects; Secondary schools; Morphology.

*Contacto: karinahess@hotmail.com

issn: 1989-0397

Recibido: $\quad 27$ de enero de 2019

www.rinace.net/riee/

https://revistas.uam.es/riee 


\section{Introducción}

En años recientes los estudios psicolingüísticos que buscan establecer relaciones entre el desarrollo lingüístico tardío, es decir, aquel que se da a partir de los seis años de edad, y el desempeño escolar han empezado a interesarse cada vez más por estudiar el desarrollo del vocabulario de los niños y adolescentes (véanse, por ejemplo, Anglin, 1993; Rojas, 2005; Schleppegrell, 2004; Snow y Uccelli, 2009; Stahl, 1999; entre otros). Lo anterior se debe a que la adquisición de léxico ha sido relacionada con el desarrollo conceptual (Rojas, 2005; Stahl, 1999), la presencia de habilidades lingüísticas complejas (Gómez, et al., 2016; Levie et al., 2016; Ravid y Geiger, 2009) y la comprensión lectora (Baumann, 2008; Carlisle, 2004; Cassany, et al., 1994; Kelley et al., 2010; Kieffer y Lesaux, 2007; MancillaMartinez y Lesaux, 2011; Marchant et al., 2007; Nippold, 2007; Ravid, 2004; Thorne et al., 2013). Por otro lado, se ha señalado que en el ámbito escolar los maestros tienden a usar el nivel de vocabulario de los alumnos para evaluar su desempeño, así como que el nivel de léxico es un predictor importante del éxito académico (Beck et al., 2008; Dockrell y Messer, 2004; Llauradó y Tolchinsky, 2013; Nagy y Townsend, 2012; St. John y Vance, 2014; Zwiers, 2008).

De acuerdo con diversos autores, durante los años escolares los niños adquieren entre 2000 y 3000 palabras por año (Ameel et al., 2011; Kieffer y Lesaux, 2007; Nippold, 2007), si bien existe una gran variabilidad en el proceso de adquisición de léxico entre individuos, puesto que hay niños que llegan a aprender hasta 5000 palabras por año, en tanto que otros no llegan ni a las 1000 (Stahl y Shiel, 1992). Hoff (2014) señala que en los primeros tres grados de la Educación Primaria los niños en general se apropian de alrededor de 9000 palabras nuevas, mientras que entre el $4^{\circ}$ y el $6^{\circ}$ grado aumentarán su vocabulario en 20,000 palabras. Esto muestra que la velocidad de adquisición de léxico incrementa conforme se avanza en escolaridad. Lo anterior se ha explicado por la relación directa que existe entre el desarrollo del vocabulario y el léxico empleado en los contenidos y materiales escolares a los que se enfrentan los estudiantes y que se complejizan conforme avanzan en los grados escolares (Anglin, 1993; Dockrell y Messer, 2004; Kieffer y Lesaux, 2007; Schleppegrell, 2004; Snow y Uccelli, 2009).

El léxico que deben adquirir los niños y adolescentes en la escuela difiere en gran medida del vocabulario que se aprende en las interacciones comunicativas cotidianas. Al respecto, Snow y Uccelli (2009) señalan que el vocabulario escolar es de tipo académico y se caracteriza por una elección léxica con palabras más diversas, de uso formal y prestigiosas, de alta precisión y que permiten hacer referencia a conceptos técnicos y abstractos, además de que son palabras de baja frecuencia en el uso cotidiano. Zwiers (2008), por su parte, afirma que el vocabulario empleado en el lenguaje académico debe tener la capacidad de describir conceptos complejos y abstractos de la manera más clara posible, así como de hacer referencia a procesos cognitivos de alto nivel. Por ello son frecuentes las palabras usadas en sentido figurado, así como las nominalizaciones. En general las palabras que los niños y adolescentes aprenden en la escuela tienden a ser más largas en términos de sílabas, menos frecuentes, de significado más especializado y de uso más formal (Hoff, 2014; Llauradó y Tolchinsky, 2013). Además, hacen referencia a conceptos menos concretos, familiares e imaginables, pues muchas veces se refieren a estados internos, cognitivos y afectivos (Ravid, 2004).

De manera adicional, es importante tomar en cuenta que el proceso de adquisición léxica no sólo se refiere a la acumulación de nuevos vocablos en el repertorio. Durante los años 
escolares los niños y adolescentes adquieren, además de las palabras mismas, todo un conocimiento sobre las características y funciones que éstas tienen como unidades comunicativas en el contexto en que aparecen (Hess, 2013, 2018; Barriga, 2002; Crystal, 1987; Dockrell y Messer, 2004; Levie et al., 2016; Nagy y Townsend, 2012; PenceTurnbull y Justice, 2008; Ravid, 2004; Zwiers, 2008). Cassany y otros (1994) señalan que el conocimiento de una palabra involucra saber cómo se pronuncia y escribe, qué morfemas la constituyen, cómo se emplea adecuadamente dentro de oraciones bien formadas, cuáles son sus acepciones y usos figurados, cómo usarla dentro de un contexto dado para conseguir un determinado propósito, cuál es su valor dialectal y de registro para emplearla con adecuación en una situación comunicativa dada y, por último, cuál es su proveniencia etimológica. Es decir, la competencia léxica incluye, como señala Baralo (2007), todo un conjunto de asociaciones fónicas, morfológicas, léxicas, semánticas, discursivas y socioculturales entre las diversas unidades constitutivas del vocabulario. De esta manera, los individuos que aprenden una palabra nueva deben adquirir además los conceptos a los que se refiere y las estrategias para usarla con eficacia y adecuación. Así, desarrollarán una red semántica organizada en la que las palabras se asocian de manera paradigmática, es decir, por jerarquía conceptual (Martín, 2018; Nippold, 2007). Debido a todo lo anterior, la adquisición del léxico académico escolar es uno de los mayores retos a los que enfrentan los niños y adolescentes durante la escolaridad primaria y secundaria (Jisa, 2004; Kieffer y Lesaux, 2007).

Si bien se ha visto que durante los años escolares existe un crecimiento importante del vocabulario en los niños y adolescentes, esto no siempre es así. Algunos estudios señalan que no todos los niños ingresan a la escuela con el mismo bagaje léxico, lo que puede representar una desventaja académica en relación con sus pares (Beck et al., 2008; Carmiol et al., 2013; Mancilla-Martínez y Lesaux, 2011; Nagy y Townsend, 2012). Los estudiantes de bajo nivel socioeconómico tienden a tener un vocabulario más restringido que sus pares de nivel socioeconómico alto y esta diferencia tiende a incrementarse conforme avanzan en su escolaridad (Anglin, 1993; Hoff, 2014; Kieffer y Lesaux, 2007; Marchand et al., 2007). Para muchos niños el vocabulario académico empleado en la escuela puede ser incluso casi tan ajeno como una lengua extranjera, por lo que acceder a los materiales escolares les resulta un reto descomunal (St. John y Vance, 2014). Es, por tanto, obligación de la escuela proporcionar a todos los estudiantes las herramientas necesarias que les permitan adquirir el vocabulario académico que los lleve a poderse desempeñar adecuadamente tanto en el ámbito escolar como más adelante en su vida laboral (Kieffer y Lesaux, 2007; Mancilla-Martinez y Lesaux, 2011; Nagy y Townsend, 2012; Rojas, 2005).

De acuerdo con Rojas (2005), la escuela tiene que proporcionar a los estudiantes una enseñanza planificada del léxico. Lo anterior se debe a que muchos niños y adolescentes tienden a tener un repertorio léxico restringido, lo que se convierte en un obstáculo para su proceso educativo, la promoción social en un futuro y el acceso a un pensamiento de orden superior. Sin embargo, las escuelas pocas veces se concentran en la enseñanza estructurada del vocabulario (Kelley et al., 2010) y los maestros emplean muy poco tiempo de sus clases para enseñar palabras (Kieffer y Lesaux, 2012). Fue, por tanto, objetivo de la presente investigación diseñar, implementar y evaluar un proyecto didáctico para la enseñanza de estrategias de adquisición de vocabulario en alumnos hispanohablantes de tercer grado de secundaria. Lo anterior resulta relevante dado que los estudios de esta índole son sumamente escasos en nuestra lengua (entre las pocas investigaciones relacionadas véanse Jaichenko y Wilson, 2013; Martín, 2018 y Rojas, 2005, 2006). 


\section{Fundamentación Teórica}

\subsection{La enseñanza del vocabulario en la escuela}

Una adecuada enseñanza de vocabulario en la escuela debe tomar en cuenta, en un primer momento, la forma en que los niños y adolescentes se apropian de nuevas palabras de manera natural y espontánea. Al respecto, Clark (2014) señala que los niños no aprenden palabras de manera aislada, sino que siempre lo hacen cuando éstas están relacionadas con otras palabras dentro de un contexto determinado. Por ello el aprendizaje de nuevas palabras se da dentro de categorías conceptuales a partir de las inferencias que los niños hacen del nuevo concepto con base en su conocimiento del mundo. De acuerdo con St. John y Vance (2014), mientras mayor conocimiento posean los niños sobre su lengua y una palabra, menor esfuerzo deberán poner en la adquisición del nuevo término, dado que se podrán basar en el conocimiento previo que tienen sobre el mismo y sus relaciones con otras palabras ya conocidas. De esta manera, el conocimiento pleno y flexible que un niño pueda adquirir sobre una palabra se desarrolla a lo largo de múltiples exposiciones y oportunidades de emplearla, puesto que cada vez que se enfrenta a una palabra acumula información sobre la misma (al respecto véase también Stahl, 1999 y Rojas, 2006). Lo anterior explica por qué los niños que tienen un vocabulario más amplio muestran una mayor facilidad y rapidez para aprender nuevas palabras que los niños con un vocabulario más restringido.

De acuerdo con Nippold (2007), existen tres maneras en las que los niños y adolescentes adquieren nuevas palabras durante los años escolares. Por un lado, pueden aprenderlas por instrucción directa, es decir, porque algún adulto o material de consulta (diccionario, glosario, enciclopedia) les proporciona la definición del término desconocido. Por otro lado, les es posible obtener el significado de palabras desconocidas con base en una abstracción contextual, que se da cuando el individuo toma en cuenta el contexto donde aparece una palabra e infiere el significado de la misma a partir de las relaciones con otros términos. Por último, los niños en edad escolar o los adolescentes pueden hacer un análisis morfológico de las palabras, es decir, descomponer una palabra desconocida en sus morfemas para extraer el significado de las partes y llegar al significado de la palabra completa. Debido a que, como se verá más adelante, el análisis morfológico es una estrategia que resulta ser muy adecuada para la adquisición de léxico académico, fue el foco del presente estudio.

Han sido diversos los investigadores que han intentado proponer lineamientos para la enseñanza del vocabulario en la escuela. De acuerdo con Alexander-Shea (2011), la enseñanza del léxico en el ámbito escolar debe contener cuatro elementos clave: 1) la activación del conocimiento previo del estudiante sobre la palabra por aprender; 2) llevar al alumno a establecer relaciones entre conceptos; 3) realizar comparaciones y contrastes entre conceptos familiares; y 4) buscar que los estudiantes generen por sí mismos el significado de la palabra desconocida. Lo anterior coincide con las propuestas de Stahl (1999), Murphy y otros (2017) y Rojas (2006), quienes señalan que toda instrucción sobre vocabulario debe incluir actividades en las que los estudiantes participen de manera activa en el aprendizaje de las palabras, puesto que está probado que los niños retienen mejor la información sobre la que han realizado operaciones cognitivas. En este sentido, los docentes no deben enseñar a sus alumnos a elaborar y memorizar listas de palabras nuevas, sino que deben proporcionarles múltiples exposiciones a información significativa sobre las palabras, así como promover discusiones en clase sobre los significados de las mismas. 
De acuerdo con Nagy y Townsend (2012), para que la enseñanza del vocabulario pueda ser efectiva se deben tratar las palabras más como una herramienta lingüística que cumple con un objetivo comunicativo real que como meros elementos que deben ser memorizados (al respecto véase también Schleppegrell, 2004).

Por otro lado, Kelley y otros (2010) señalan que toda instrucción de vocabulario en la escuela debe seguir tres principios:

1. Enfocar la enseñanza al conocimiento profundo de un número relativamente pequeño de palabras, sus elementos y relaciones semánticas dentro de contextos ricos.

2. Llevar a cabo una elección de las palabras que se busca enseñar con la finalidad de que sean de alta utilidad para los estudiantes.

3. Equilibrar la enseñanza directa de palabras con la enseñanza de estrategias para aprender palabras.

Un aspecto que toda enseñanza de vocabulario debe tomar en consideración es que resulta imposible que los maestros proporcionen una instrucción específica para todas las palabras que deben aprender los estudiantes durante su paso por la escuela (St. John y Vance, 2014), sobre todo si se sabe que un alumno promedio de educación superior debe llegar a tener un vocabulario de alrededor de 45,000 palabras y en la escuela solo es posible enseñar entre 300 y 500 palabras al año (Stahl, 1999; Stahl y Shiel, 1992). Por lo anterior, se ha propuesto que, además de la enseñanza de ciertas palabras esenciales, los maestros proporcionen una instrucción planeada de estrategias para aprender palabras nuevas (Baumann, 2008; Bauman et al., 2007; Cassany et al. 1994; Graves, 2006; Kieffer y Lesaux, 2007; Stahl, 1999). Al enseñar estrategias para la adquisición de léxico, se le proporcionan al estudiante las herramientas necesarias para que pueda llevar a cabo un aprendizaje independiente de las palabras nuevas a las que se enfrenta (Kieffer y Lesaux, 2007).

Una de las propuestas nodales para la enseñanza de estrategias para la adquisición de vocabulario es la de Graves (2006). De acuerdo con este autor, la enseñanza de estrategias para inferir el significado de los términos les permite a los estudiantes duplicar la cantidad de palabras que aprenden. Bajo este enfoque, se enseña a los alumnos a reconocer que existen muchas maneras de aprender palabras y que es posible adquirir estrategias que permiten el aprendizaje del nuevo léxico. Además, se busca que los estudiantes se den cuenta de que aprender palabras es importante, con la finalidad de que se vuelvan responsables en la construcción de su propio vocabulario y se comprometan personalmente con su aprendizaje. Para lograr todo lo anterior, Graves (2006) propone que se enseñe a los alumnos cada estrategia, así como de qué manera y en qué situación debe emplearse. Esto implica un modelamiento por parte del maestro, un aprendizaje activo de la estrategia por parte del alumno (mediante un uso colaborativo con pares, prácticas guiadas) y finalmente el uso independiente de la estrategia. Además, Graves menciona que es esencial que los maestros expliciten el valor de las estrategias al decirles a los alumnos por qué es importante que las aprendan y para qué les van a servir en su desempeño académico y en su vida futura. Finalmente, las estrategias deben incentivar en todo momento la reflexión sobre las palabras con el objeto de que los estudiantes puedan planear su aprendizaje. 
El programa de enseñanza de vocabulario de Graves (2006) propone cinco estrategias centrales para que los alumnos se conviertan en aprendices competentes de nuevas palabras:

1. Aprender a usar el contexto para descubrir el significado de las palabras desconocidas dentro de experiencias ricas y variadas con el lenguaje (lectura en voz alta por parte del maestro, lectura independiente de los alumnos, discusiones literarias, actividades de escritura).

2. Emplear las partes de las palabras (morfemas) para hallar el significado de las palabras desconocidas (pistas para descomponer palabras morfológicamente complejas e inferir el significado de la palabra completa).

3. Aprender a usar el diccionario y otras herramientas de consulta.

4. Desarrollar una estrategia para enfrentarse a palabras desconocidas (uso del contexto y/o descomposición de las palabras).

5. Adoptar un acercamiento personal a la construcción del propio vocabulario (fomento al interés por aprender palabras mediante juegos con lenguaje y la reflexión metalingüística sobre las mismas).

Las estrategias propuestas por Graves (2006) han sido probadas por otros investigadores (Baumann, 2008; Bauman, et al., 2007) en programas de instrucción de vocabulario con alumnos angloparlantes y se ha observado que los estudiantes que aprendieron a usar las estrategias muestran un incremento importante en su nivel de vocabulario, además de que empiezan a emplear palabras más sofisticadas en sus textos escritos, incrementan su interés por aprender palabras nuevas y hacen un uso independiente de las estrategias incluso meses después de la intervención didáctica. Por tanto, es posible afirmar que los alumnos efectivamente se pueden beneficiar de la enseñanza de estrategias de aprendizaje de vocabulario para adquirir palabras de manera independiente. En este sentido, la presente investigación se propuso crear un proyecto didáctico para la enseñanza de estrategias de análisis morfológico para aprender vocabulario en estudiantes hispanohablantes que tomara en cuenta tres de las estrategias propuestas por Graves (2006): la descomposición morfológica, el uso del diccionario y la adopción de un acercamiento personal para la construcción del propio vocabulario (puntos 2,3 y 5 , respectivamente).

\subsection{La enseñanza de estrategias de análisis morfológico para aprender nuevas palabras}

Como se vio anteriormente, una de las habilidades lingüísticas principales que permite la adquisición de vocabulario durante los años escolares es el análisis morfológico de las palabras, es decir, el saber que las palabras pueden estar formadas por elementos con significado (morfemas) que se combinan para generar un significado general (Hoff, 2014; Levie et al., 2016; Nippold, 2007; Sparks y Deacon, 2015). Una vez que los niños aprenden a analizar las palabras en sus morfemas constituyentes, a asignar un significado a las partes y ver qué combinaciones son posibles en la lengua que adquieren, se vuelven capaces de emplear las raíces, sufijos y prefijos derivativos para denotar nuevos significados (Clark, 2009; Goodwin et al., 2017).

Para realizar un análisis de las palabras morfológicamente complejas el individuo debe identificar los morfemas que constituyen la palabra, acceder al significado de cada uno, 
determinar de qué manera los afijos cambian el significado de la raíz y, por último, combinar las piezas informativas para encontrar el significado de la palabra completa (Larsen y Nippold, 2007). Al respecto, Kieffer y Lesaux (2012) señalan que la conciencia morfológica sobre las palabras implica la habilidad para reconocer los morfemas de las mismas y su relación semántica con las diferentes formas morfológicas, conocer las posibles funciones gramaticales de los morfemas dentro de la palabra y además saber de qué manera se distribuyen los morfemas en la lengua que se adquiere. De esta manera, cuando el aprendiz de un término se enfrenta a una palabra desconocida la divide en sus partes con significado (morfemas), establece hipótesis sobre el significado de la palabra completa y revisa que dicho significado sea el adecuado de acuerdo con el contexto donde aparece y con base en los conocimientos previos que tenga sobre la misma. Así es como se explica que un aprendiz eficiente de palabras nuevas pueda emplear su conocimiento sobre raíces frecuentes para acceder al significado de otras palabras de poca frecuencia.

La capacidad para realizar un análisis morfológico de las palabras inicia desde la edad preescolar (Auza, 2005; 2006), se desarrolla hasta muy entrada la adolescencia (Anglin, 1993; Hess, 2018; Larsen y Nippold, 2007; Sparks y Deacon, 2015) y continúa su evolución incluso en la adultez (Tighe y Binder, 2015). Lo anterior se debe a que requiere de un procesamiento tanto de la forma como del significado de las palabras (Carlisle, 2004). Diversos autores han señalado que la capacidad para analizar morfológicamente las palabras muestra un incremento muy importante alrededor del $5^{\circ}$ grado y que conforme crecen los individuos son cada vez más capaces de hacer reflexiones explícitas sobre los morfemas que componen las palabras (Anglin, 1993; Hess, 2018; Carlisle, 2004; Graves, 2006; Kieffer y Lesaux, 2007; Larsen y Nippold, 2007; Nippold y Sun, 2008; Tighe y Binder, 2015). Nippold y Sun (2008) afirman que el crecimiento durante los años escolares de la capacidad para analizar los morfemas de las palabras se debe principalmente a que las palabras morfológicamente complejas ocurren muy frecuentemente en los materiales académicos e incrementan su aparición conforme avanzan los grados escolares (al respecto véase también Carlisle, 2004, Kieffer y Lesaux, 2007; Levie et al., 2016, y Tighe y Binder, 2015).

Por otra parte, se ha señalado que existe una estrecha relación entre la capacidad para hacer un análisis morfológico de las palabras y la comprensión lectora (Bangs y Binder, 2016; Bowers et al., 2010; Carlisle, 2004; De Freitas et al., 2018; Goodwin et al., 2017; Jaichenco y Wilson, 2013; Kieffer y Lesaux, 2012; Mahony et al., 2000; Nippold y Sun, 2008; Tighe y Binder, 2015). De acuerdo con Li y Wu (2015), la conciencia morfológica sobre las palabras permite aumentar la velocidad de acceso a las mismas, lo que mejora la comprensión lectora tanto en lenguas con escritura alfabética como en aquellas con escritura no alfabética (al respecto véase también De Freitas, et al., 2018 y Jaichenco y Wilson, 2013). Además, se ha observado que la relación entre la capacidad para hacer un análisis morfológico y la comprensión lectora incrementa conforme aumentan los grados escolares (Bangs y Binder, 2016; Li y Wu, 2015; Ravid y Geiger, 2009).

La enseñanza en la escuela de estrategias de análisis morfológico para aprender nuevas palabras resulta entonces fundamental por diversas razones. Por un lado, es la segunda estrategia más importante para aprender palabras nuevas después de la abstracción contextual y tiene la ventaja de que puede ser enseñada directamente (Graves, 2006). Además, se sabe que entre el 60 y el $80 \%$ de las palabras nuevas a las que se enfrentan los estudiantes en la escuela son palabras morfológicamente complejas (Kieffer y Lesaux, 2012; Larsen y Nippold, 2007; Pacheco y Goodwin, 2013; Sparks y Deacon, 2015) y que 
dichas palabras representan ideas abstractas importantes que dan cuenta de contenidos académicos esenciales que los alumnos deben adquirir (Kieffer y Lesaux, 2012; Pacheco y Goodwin, 2013; Nippold y Sun, 2008). También se ha visto que en lugar de memorizar una gran cantidad de palabras del vocabulario complejo contenido en los textos académicos los alumnos pueden emplear sus conocimientos morfológicos para comprender en promedio tres palabras adicionales para cada palabra nueva aprendida (Pacheco y Goodwin, 2013). Asimismo, diversos estudios muestran que gran parte de los estudiantes preparatorianos no está consciente de que las palabras pueden descomponerse para obtener su significado (Stahl, 1999; Stahl y Shiel, 1992). Por otro lado, hay evidencia de que si bien los niños emplean diversas estrategias para extraer el significado de las palabras desconocidas -como el contexto-, les resulta más eficaz la estrategia de análisis morfológico cuando conocen la raíz de la palabra nueva (Larsen y Nippold, 2007). Finalmente, se ha visto que la enseñanza de estrategias de análisis morfológico tiene efectos muy favorables en la conciencia morfológica y la compresión lectora de estudiantes de bajo rendimiento (Bowers, et al., 2010; Kieffer y Lesaux, 2012; Stahl, 1999) porque la capacidad para descomponer palabras en sus morfemas es, al lado de la conciencia fonológica (al respecto véase Gutiérrez y Díez, 2018), esencial para que el individuo se convierta en un buen lector $\mathrm{y}$, por tanto, para que tenga un adecuado desempeño académico (Corrêa y Peruzzi, 2018; De Freitas, Mota y Deacon, 2018; Jaichenko y Wilson, 2013; Mahony, Singson y Mann, 2000; Ramachandra y Karanth, 2007; Ravid y Geiger, 2009).

\section{Objetivos}

La presente investigación tuvo los siguientes objetivos:

- Diseñar e implementar un proyecto didáctico para la enseñanza de estrategias de análisis morfológico de palabras para incrementar las habilidades para aprender vocabulario en alumnos hispanohablantes de tercer grado de secundaria.

- Evaluar si el proyecto didáctico mejora las habilidades de los alumnos para segmentar las palabras en sus morfemas.

- Evaluar si el proyecto didáctico incrementa las habilidades de los alumnos para encontrar el significado de las palabras a través del significado de sus morfemas.

\section{Método}

\subsection{Participantes}

Formaron parte del proyecto de investigación 38 estudiantes de tercer grado de secundaria de una escuela particular de la ciudad de Santiago de Querétaro, México. El grupo experimental (19 estudiantes) se enfrentó al proyecto didáctico foco de la investigación. En cambio, el grupo control (19 alumnos) participó en un proyecto didáctico sobre la diversidad lingüística en el mundo, en el que se trabajó la comprensión y producción de textos informativos y la lectura e interpretación de tablas y gráficos. Ambos proyectos fueron llevados a cabo en diez sesiones de 45 minutos cada una a lo largo de cuatro semanas. 


\subsection{Descripción del proyecto didáctico}

Con base en los antecedentes señalados, el proyecto didáctico fue diseñado con la finalidad de:

1. Encaminar a los estudiantes hacia una participación activa en la construcción de los significados de las palabras mediante actividades de resolución de problemas léxicos que los llevaran a generar por sí mismos el significado de los términos desconocidos;

2. Conducir a los alumnos a que establecieran relaciones entre palabras (analogías) por medio de comparaciones y contrastes entre conceptos nuevos y familiares;

3. Motivar a los estudiantes para que se interesaran por aprender palabras nuevas; $\mathrm{y}$

4. Fomentar la reflexión sobre las palabras: sus significados, usos y distribución.

Específicamente en el caso de la enseñanza de estrategias para el análisis morfológico de las palabras se buscaba:

1. Mostrar a los alumnos la estructura de las palabras morfológicamente complejas;

2. Llevar a los alumnos a identificar las raíces de las palabras y a descubrir las reglas y patrones de formación de las mismas (uso de prefijos y sufijos);

3. Enseñar a los estudiantes diversas estrategias de composición y descomposición de palabras, así como de resolución de problemas morfológicos (estrategias para ir de las partes al todo, del todo a las partes y para establecer analogías con la finalidad de llegar al significado de las palabras);

4. Enseñar raíces, prefijos y sufijos grecolatinos frecuentes en el español que les permitieran aprender nuevas palabras;

5. Hacer que los estudiantes descubrieran la categoría gramatical a la que pertenece una palabra así como el papel que juegan los sufijos para cambiar las categorías gramaticales de los términos; y

6. Llevar a los alumnos a reflexionar sobre los casos donde los significados de las palabras no se desprenden directamente de la suma de los morfemas porque en su formación intervienen usos históricos (p.ej., sal-salario) o metafóricos (p. ej., alumbrar-lumbrera) de las raíces.

Por otra parte, en lo que se refiere a la enseñanza del uso del diccionario el proyecto didáctico buscó que los estudiantes:

1. Conocieran la estructura textual y las características generales del diccionario;

2. Se percataran de que las palabras definidas pueden tener más de una acepción y que algunas acepciones pueden ser de carácter no literal; y

3. Observaran que las definiciones incluyen la categoría gramatical de la palabra.

Finalmente, para propiciar que los alumnos se motivaran a aprender nuevas palabras y adoptaran un acercamiento personal a la construcción de su propio vocabulario se llevó a los estudiantes a que: 
1. Participaran en actividades interesantes que les presentaran retos cognitivos que los condujeran a descubrir los significados de las palabras desconocidas;

2. Realizaran juegos lingüísticos con morfemas, palabras y significados; y

3. Adoptaran una actitud creativa en la formación de palabras y significados al participar en interacciones humorísticas con las palabras que los llevaran a disfrutar su aprendizaje.

Para lograr un proyecto didáctico que cumpliera con todos los objetivos anteriormente descritos, se propuso a los estudiantes la elaboración de un Diccionario de Palabras Inventadas (véase la descripción del proyecto en el cuadro 1).

Cuadro 1. Descripción de las sesiones del proyecto didáctico

\begin{tabular}{|c|c|}
\hline SESIÓN & DESCRIPCIÓN \\
\hline 1 & $\begin{array}{l}\text { Introducción al proyecto: presentación de una muestra de un Diccionario de Palabras } \\
\text { Inventadas; análisis del diccionario como portador textual; características del } \\
\text { diccionario; discusión grupal sobre las etimologías grecolatinas y su valor en nuestra } \\
\text { lengua } \\
\text { Lectura del texto "Entérate: Las } 15 \text { fobias más extrañas". Por parejas segmentar y } \\
\text { analizar el significado de las palabras correspondientes a las fobias (palabras Raíz- } \\
\text { Raíz) }\end{array}$ \\
\hline 2 & $\begin{array}{l}\text { Descubrir que las palabras formadas con etimologías constan de raíces con sufijos y/o } \\
\text { prefijos. } \\
\text { Analizar listas de palabras que poseen prefijos vs palabras que no los poseen (Ejem. } i \text { - } \\
\text { lógico vs. iglesia). Ejercicios diversos con prefijos. }\end{array}$ \\
\hline 3 & $\begin{array}{l}\text { Extraer el significado de una raíz con base en una serie de palabras que la contienen. } \\
\text { Ejem: ORTO en ortografía, ortodoncia, ortopedia, ortodoxo }\end{array}$ \\
\hline 4 & $\begin{array}{l}\text { Análisis de sufijos: segmentación, identificación del significado, descubrimiento de su } \\
\text { valor para el cambio de categoría gramatical (Ejem: fermento/fermenta-ción) }\end{array}$ \\
\hline 5 y 6 & $\begin{array}{l}\text { Obtener el significado de una raíz aislada a través de analogías siguiendo los siguientes } \\
\text { pasos: } \\
\text { 1) buscar dos o tres palabras conocidas que contengan la raíz } \\
\text { 2) ver cómo cambia la raíz cuando se le agregan morfemas (otra raíz, prefijo o sufijo) } \\
\text { 3) extraer el significado de los morfemas añadidos } \\
\text { 4) realizar comparaciones entre las palabras para extraer el significado de la raíz } \\
\text { buscada }\end{array}$ \\
\hline 7 y 8 & $\begin{array}{l}\text { Elaborar por parejas } 20 \text { palabras inventadas para el diccionario. Para cada palabra } \\
\text { indicar: } \\
\text { 1) término inventado } \\
\text { 2) categoría gramatical (sustantivo, adjetivo, verbo, adverbio) } \\
\text { 3) definición literal } \\
\text { 4) definición metafórica (juego con lenguaje) }\end{array}$ \\
\hline 9 & $\begin{array}{l}\text { Revisión por pares de las palabas inventadas. Corrección de los señalamientos de los } \\
\text { pares y entrega al maestro para revisión }\end{array}$ \\
\hline 10 & Revisión de los señalamientos del maestro \\
\hline
\end{tabular}

Fuente: Elaboración propia.

Después de diversas sesiones de trabajo en torno a las características textuales del diccionario y el análisis morfológico de palabras (segmentación de morfemas y establecimiento de analogías para descubrir el significado de morfemas y palabras), en parejas los alumnos tuvieron que inventar palabras que se incluirían en un diccionario que contemplaría todos los términos inventados por el grupo y que todos los estudiantes se llevarían de regalo al terminar el proyecto. Para crear las palabras, los alumnos podían 
hacer uso de las palabras y morfemas vistos durante la realización de los ejercicios del proyecto didáctico y con los cuales habían elaborado un glosario, además de que se les proporcionaban diccionarios etimológicos del español que podían consultar si lo deseaban. Se les indicó a los alumnos que las palabras inventadas debían cumplir con las siguientes características: a) ser una palabra que no existiera; b) estar formadas exclusivamente por etimologías grecolatinas, b) constar de al menos dos morfemas y c) que respetaran la fonología española (p. ej., oropié vs. aurumpedis). Para cada palabra los estudiantes debían incluir la categoría gramatical y una definición literal. Con el fin de que los alumnos se dieran cuenta de que las palabras pueden tener más de un significado y que muchas veces éste es de tipo no literal, se les incitó a que jugaran con las palabras para además proporcionar para cada término una segunda acepción de tipo metafórica. En la figura 1 puede observarse un ejemplo de palabras inventadas por los alumnos durante el proceso de elaboración del diccionario:

A lo largo del proyecto se cuidó que no hubiera una enseñanza explícita de los morfemas y palabras incluidas en el instrumento de evaluación (pre y pos-test), con la finalidad de poder observar si los cambios por la intervención didáctica obedecían realmente al uso de las estrategias de análisis morfológico enseñadas durante el proyecto y no al mero aprendizaje memorístico de ciertos morfemas y palabras.

\subsection{Instrumento}

Para observar si la aplicación del proyecto didáctico tenía incidencia en la manera en que los estudiantes se enfrentan a palabras morfológicamente complejas para segmentarlas en sus morfemas y extraer su significado, se aplicaron un pre-test y un pos-test con un instrumento que constó de una lista de 27 sustantivos morfológicamente complejos obtenidos a partir de un muestreo de palabras incluidas en los libros de texto de Ciencias Naturales y Ciencias Sociales oficiales de la Secretaría de Educación Pública de $5^{\circ}$ grado de primaria y autorizados por dicha institución para $1^{\circ}$ y $3^{\circ}$ de secundaria. Los 27 términos del instrumento corresponden a sustantivos de cuatro tipos: prefijo-raíz (Ejem: re-forma), raíz-sufijo (Ejem: fermenta-ción), raíz-raíz (Ejem: Arido-américa) y polimorfémicos (Ejem: in-toxica-ción) en su frecuencia de aparición real en los libros de texto (prefijo-raíz: 7,6\%, raíz-sufijo: 40,6\%, raíz-raíz: 21,8\%, polimorfémicos: 27,9\%). Para una descripción más detallada sobre las decisiones metodológicas llevadas a cabo para la creación del instrumento véase Hess (2018). Con el objeto de evitar efectos de cansancio o aprendizaje de las palabras durante la aplicación del instrumento, se elaboraron dos listas diferentes y éstas fueron contrabalanceadas para ambos grupos en el pre-test y el pos-test. El instrumento se aplicó de manera grupal. En el Apéndice se incluye un ejemplo del instrumento. 


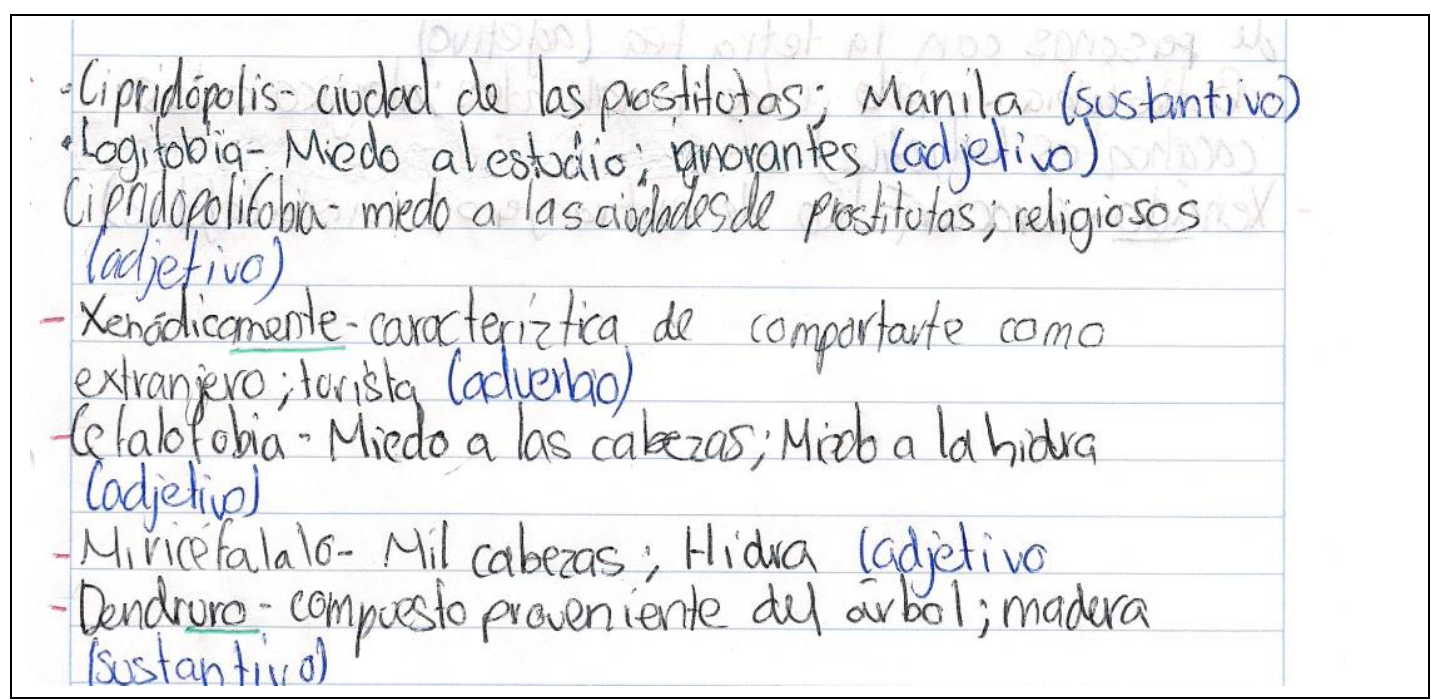

Figura 1. Ejemplo de algunas de las palabras inventadas por una pareja de estudiantes durante la elaboración del Diccionario de Palabras Inventadas

Fuente: Elaboración propia.

\subsection{Análisis de datos}

Los resultados obtenidos por los participantes de los grupos control y experimental durante el pre-test y pos-test se analizaron con base en dos aspectos: 1) capacidad para segmentar las palabras en sus morfemas y 2) obtención del significado de morfemas y palabras. Para el análisis de la segmentación de las palabras en sus morfemas las respuestas de los participantes se clasificaron en las categorías que pueden observarse en el cuadro 2 (la columna de puntaje indica la calificación otorgada a cada tipo de respuesta para el análisis estadístico)

Para observar el posible efecto de la secuencia didáctica en la habilidad de los alumnos para encontrar el significado de las palabras, se buscó ver si eran capaces de construir el significado de las mismas a partir de los morfemas, por lo que las respuestas fueron clasificadas como se muestra en el cuadro 3 (la columna de puntaje indica la calificación otorgada a cada tipo de respuesta para el análisis estadístico):

Cuadro 2. Clasificación de las respuestas de los estudiantes para la segmentación de palabras

\begin{tabular}{|c|c|c|c|}
\hline CódIGo & TIPO DE RESPUESTA & EJEMPLO & Puntaje \\
\hline A & Segmentación correcta & Fermenta/ción & 2 \\
\hline B & $\begin{array}{l}\text { Segmentación incorrecta pero recupera } \\
\text { al menos un morfema correctamente }\end{array}$ & Fer/menta/ción & 1 \\
\hline $\mathrm{C}$ & $\begin{array}{c}\text { Segmentación silábica } \\
\text { Segmentación asistemática } \\
\text { No segmenta }\end{array}$ & $\begin{array}{l}\text { Fer/men/ta/ción } \\
\text { Fer/me/ntac/ión } \\
\text { Fermentación }\end{array}$ & $\mathrm{O}$ \\
\hline
\end{tabular}

Fuente: Elaboración propia. 
Cuadro 3. Clasificación de las respuestas de los participantes para la obtención del significado de morfemas y palabras

\begin{tabular}{ccc}
\hline Código & TIPO DE RESPUESTA & PUNTAJE \\
\hline $\mathrm{X}$ & Recupera el significado de la palabra y los morfemas & 2 \\
$\mathrm{Y}$ & Recupera el significado de la palabra completa o de \\
algún morfema & 1 \\
$\mathrm{Z}$ & No recupera el significado de la palabra completa ni de \\
ningún morfema & 0 \\
\hline
\end{tabular}

Fuente: Elaboración propia.

\section{Resultados}

\subsection{Resultados generales}

Un análisis inicial mediante una prueba de $t$ de Student de las medias obtenidas a través de los puntajes otorgados a cada tipo de respuesta mostró que los grupos experimental y control eran similares entre sí antes de la intervención didáctica en la capacidad para segmentar palabras en sus morfemas $(\mathrm{t}=, 163$, g.l.=36, n.s. $=, 871)$ y en la obtención del significado de morfemas y palabras $(\mathrm{t}=1,782$, g.l. $=36$, n.s. $=, 83$ ) (para las medias y desviaciones estándar véase la Tabla 4). Lo anterior indica que los grupos eran comparables entre sí. El análisis estadístico al concluir el proyecto didáctico hizo evidente diferencias significativas entre los grupos experimental y control para los dos factores medidos: segmentación de palabras $(\mathrm{t}=-2,814$, g.l.=36, n.s. $=, 008)$ y significado de morfemas y palabras $(\mathrm{t}=-4,160$, g.l. $=36$, n.s. $=, 000)$. Lo anterior muestra que el proyecto didáctico tuvo una incidencia importante en el desarrollo de habilidades de análisis morfológico de los estudiantes del grupo experimental.

Cuadro 4. Medias y desviaciones estándar a partir de los puntajes alcanzados en pre y postest para las tareas de segmentación de palabras y obtención del significado de morfemas y palabras para ambos grupos

\begin{tabular}{cllllc}
\hline \multirow{2}{*}{ TAREA } & GruPo & N & MEDIA & $\begin{array}{c}\text { DESVIACIÓN } \\
\text { ESTÁNDAR }\end{array}$ \\
\hline \multirow{3}{*}{ Pre-test } & \multirow{2}{*}{ Segmentación } & Experimental & 19 & $3, .8947$ & 4,21498 \\
& \multirow{2}{*}{ Significado } & Control & 19 & 32,1579 & 5,63977 \\
& Experimental & 19 & 26,0000 & 5,99073 \\
& \multirow{2}{*}{ Pegmentación } & Control & 19 & 30,2105 & 8,37708 \\
\cline { 3 - 5 } & \multirow{2}{*}{ Significado } & Experimental & 19 & 36,7368 & 3,01555 \\
& Control & 19 & 32,7895 & 5,31851 \\
& Experimental & 19 & 35,5789 & 5,33662 \\
& Control & 19 & 28,3158 & 5,42681 \\
\hline
\end{tabular}

Fuente: Elaboración propia.

\subsection{Resultados entre pre y pos-test para la segmentación de palabras}

Una comparación entre pre y pos-test de la capacidad de los alumnos de los grupos experimental y control para segmentar las palabras en sus morfemas puede observarse en las figuras 2 y 3 , respectivamente. 


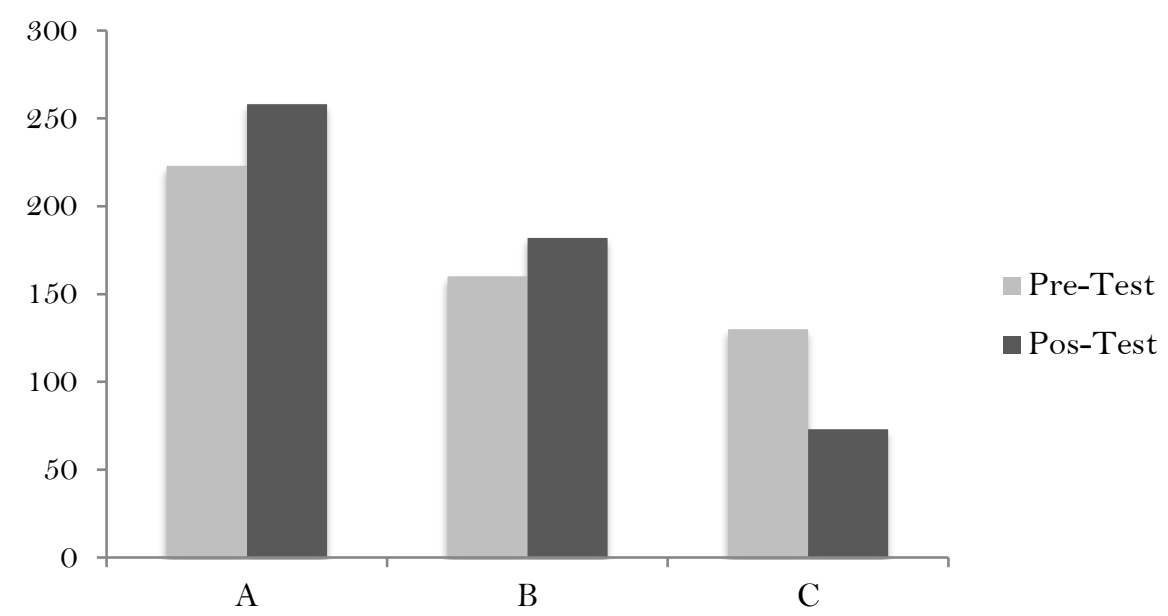

Figura 2. Cantidad total de respuestas por tipo para la segmentación de palabras en el grupo experimental

$\mathrm{El}$ eje de la " $\mathrm{x}$ " muestra el tipo de respuesta $(\mathrm{A}=$ segmentación correcta, $\mathrm{B}=$ segmentación incorrecta, pero recupera al menos un morfema correctamente, $\mathrm{C}=$ segmentación asistemática, silábica o no segmenta) y el eje de la "y" indica el número de respuestas. El total de respuestas posibles era 1026.

Fuente: Elaboración propia.

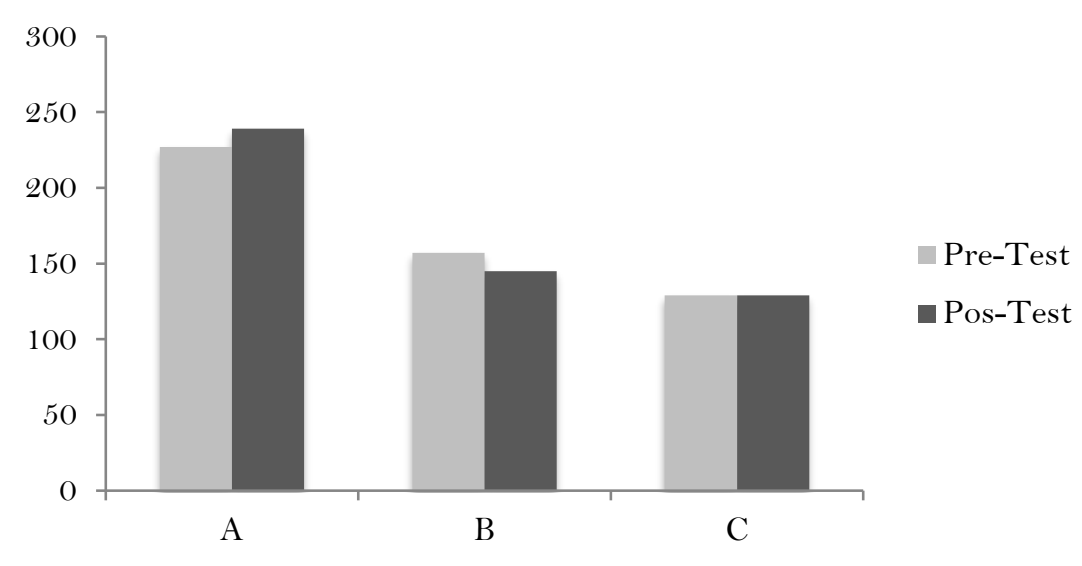

Figura 3. Cantidad total de respuestas por tipo para la segmentación de palabras en el grupo control

Fuente: Elaboración propia.

Como puede apreciarse en las figuras anteriores, en el grupo experimental hay un crecimiento de las respuestas tipo A (segmentación correcta) y B (segmentación incorrecta pero segmenta al menos un morfema correctamente), así como una disminución de las respuestas tipo C (segmentación asistemática, silábica o no segmenta). Dicho cambio entre pre y pos-test resultó ser estadísticamente significativo mediante el análisis de medias con una prueba de $t$ de Student ( $\mathrm{t}=-5,822$, g.l. $=18$, n.s. $=, 000$ ). En cambio, el grupo control no mostró variación importante en el tipo de respuestas de segmentación de palabras, lo que se confirmó estadísticamente (t=-,502., g.l.=18, n.s.=,622). 


\subsection{Resultados entre pre y pos-test para la obtención del significado de palabras.}

A continuación se procedió a analizar los resultados correspondientes al pre y pos-test en la obtención del significado de las palabras y morfemas. Los resultados se observan en las figuras 4 (grupo experimental) y 5 (grupo control):

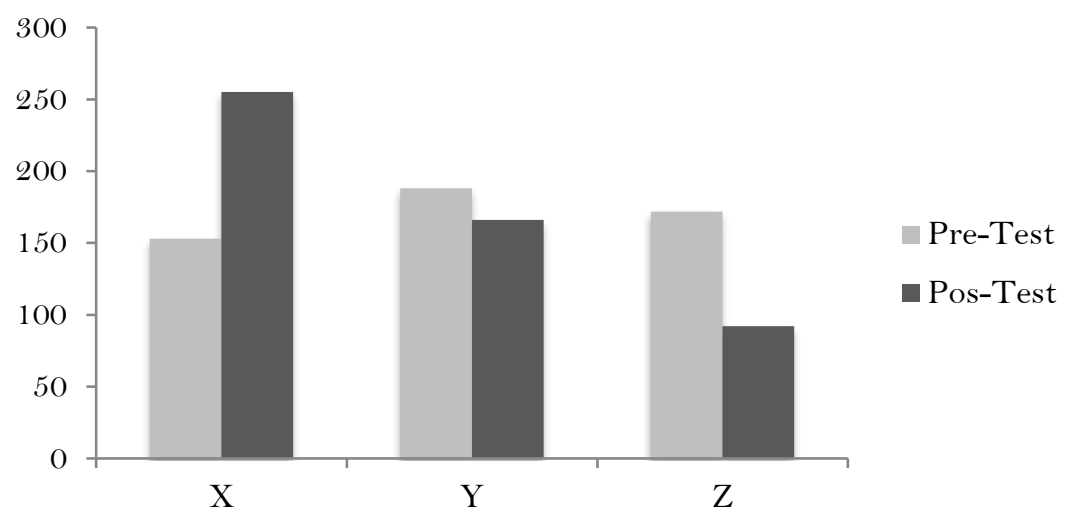

Figura 4. Cantidad total de respuestas por tipo para la obtención del significado de palabras en el grupo experimental Fuente: Elaboración propia.

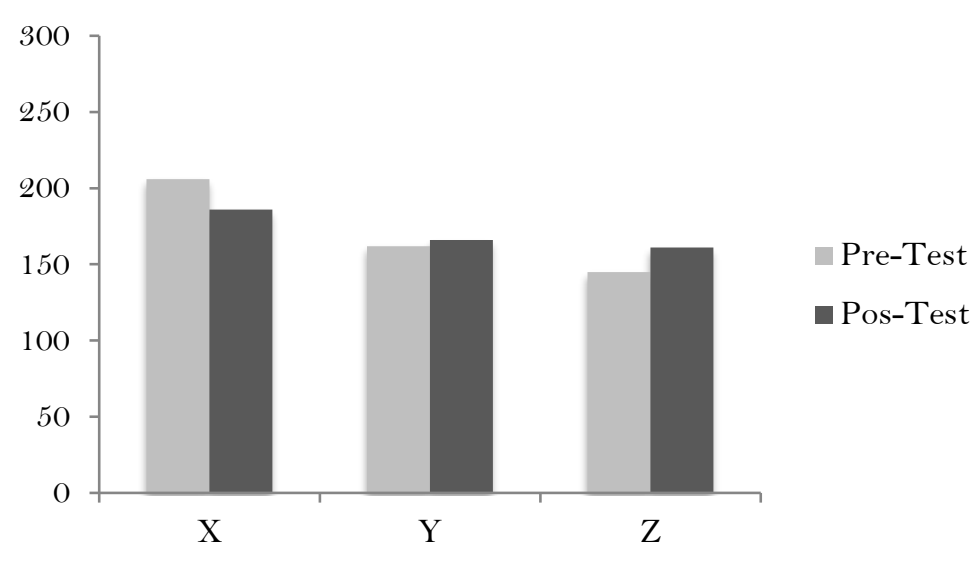

Figura 5. Cantidad total de respuestas por tipo para la obtención del significado de palabras

Fuente: Elaboración propia.

En la figura 4 es posible observar para el grupo experimental un incremento importante entre pre y pos-test en las respuestas tipo X (recupera el significado de la palabra y los morfemas) a raíz de la disminución de respuestas tipo Y (recupera el significado de la palabra o de algún morfema) y $\mathrm{Z}$ (no recupera el significado de la palabra ni de ningún morfema). Este incremento resultó ser estadísticamente significativo mediante un análisis de medias con una prueba $t$ de Student $(\mathrm{t}=-8,597$, g.l. $=18$, n.s. $=, 000)$. Por otra parte, como se observa en la Fig. 5, no hubo diferencias importantes entre pre y pos-test en el tipo de respuestas que presentaron los estudiantes del grupo control, lo que se confirmó estadísticamente ( $\mathrm{t}=1,334$, g.l.=18, n.s.=,199). 


\section{Discusión y Conclusiones}

El presente estudio tuvo como objetivo diseñar, implementar y evaluar un proyecto didáctico encaminado a la enseñanza de estrategias de análisis morfológico de palabras para la adquisición de nuevos términos en estudiantes de tercer grado de secundaria. A diferencia de otros métodos de enseñanza de vocabulario que plantean la instrucción directa de las palabras, este proyecto pretendió darle a los estudiantes herramientas que les permitieran aprender términos de manera independiente en el futuro. Mediante la elaboración de un Diccionario de Palabras Inventadas se buscó que los alumnos pensaran sobre las palabras, conocieran la manera en que éstas se estructuran, analizaran los morfemas que las constituyen, las compararan y contrastaran, aunque también que se divirtieran con la creación de nuevas palabras y significados tanto literales como no literales y mostraran un interés por construir su propio léxico.

Los resultados de la evaluación del proyecto didáctico mostraron que los estudiantes que se enfrentaron a la intervención, a diferencia de los alumnos del grupo control, tuvieron un incremento significativo en la capacidad para encontrar los morfemas que se encuentran en las palabras (respuestas tipo A y B). Además, se evidenció una disminución notable de las respuestas tipo $\mathrm{C}$ (segmentación asistemática, silábica o no segmenta). El hecho de que los alumnos del grupo experimental hayan sido capaces de encontrar con mayor facilidad los morfemas de las palabras y hayan disminuido las segmentaciones asistemáticas o silábicas indica que la intervención didáctica tuvo un efecto sustancial en su capacidad para percatarse de que los morfemas son unidades mínimas de significado que se combinan con otras unidades para formar palabras.

Por otro lado, los resultados del proyecto didáctico en relación con la obtención del significado de palabras también son muy alentadores. Los datos de nuestro estudio muestran que después de la intervención los alumnos del grupo experimental lograron recuperar el significado de casi el doble de palabras que antes del proyecto (255 vs. 153 en las respuestas tipo X). A su vez, se redujeron casi a la mitad las respuestas en las que los estudiantes no eran capaces de encontrar el significado de la palabra o de algún morfema (92 vs. 172 respuestas de tipo Z). Por tanto, existe evidencia de que las estrategias de análisis morfológico que adquirieron los alumnos durante el proyecto incidieron positivamente en su capacidad para recuperar el significado de palabras que les son desconocidas. Lo anterior coincide con lo señalado en estudios previos con estudiantes angloparlantes de nivel primaria (Baumann, 2009; Baumann, et al., 2007; Kelley, et al., 2010; Kieffer y Lesaux, 2012), secundaria (Murphy, et al., 2017; Pacheco y Goodwin, 2013) y adultos (Bangs y Binder, 2016).

$\mathrm{Si}$ bien los resultados de este estudio muestran un crecimiento importante en las habilidades de análisis morfológico de los alumnos, esto no necesariamente significa que este incremento realmente incida de manera positiva en su desempeño académico. Resulta por tanto necesario llevar a cabo más estudios que permitan observar la posible incidencia de un proyecto de análisis morfológico de palabras como el que aquí se presenta en la comprensión lectora de los estudiantes. De igual manera, sería importante realizar investigaciones donde, además de trabajar el desarrollo de estrategias de descomposición morfológica para aprender vocabulario, se complementara la enseñanza con actividades en diversos contextos comunicativos con una finalidad pragmática real que les dieran a los alumnos experiencias ricas y variadas con el lenguaje y que les permitiera la adquisición de vocabulario de una manera más natural. Adicionalmente, sería importante 
realizar estudios comparativos con niños de otros grados escolares y otros tipos de escuela para corroborar los hallazgos del presente estudio.

Consideramos que el proyecto didáctico descrito en este estudio representa una aportación importante a las escasas investigaciones sobre la enseñanza de léxico en la escuela en poblaciones hispanohablantes. Además de que la intervención didáctica mostró que es posible enseñar a los alumnos a descomponer las palabras en sus morfemas para obtener el significado de términos desconocidos, dotó a los estudiantes de enseñanzas adicionales. Aprendieron para qué sirve un diccionario, cómo se construye, así como que existen personas dedicadas a su elaboración y revisión constantes. Además, se dieron cuenta de que las palabras son importantes, que se pueden inventar, que las sociedades las crean constantemente, que se puede jugar con ellas, que tienen una historia, que se escriben de cierta manera porque provienen de otras, que pueden denotar muchos significados, que pertenecen a categorías gramaticales. El proyecto les permitió descubrir que los significados de las palabras se construyen y que hacer un esfuerzo importante por descubrir lo que significan puede resultar muy gratificante. En ese sentido, el proyecto didáctico fue significativo y motivante para los alumnos y les permitió pensar sobre las palabras y disfrutar su aprendizaje.

\section{Referencias}

Alexander-Shea, A. (2011). Redefining vocabulary: the new learning strategy for social studies. The Social Studies, 102, 95-103. https://doi.org/10.1080/00377996.2010.509371

Ameel, E., Storms, G. y Malt, B. C. (2011). Mowgli in the jungle of words: comprehension and later lexical development. En L. Carlson, C. Hoeschler, y T.F. Shipley (Eds.), Proceedings of the 33th Annual Conference of the Cognitive Science Society (pp. 2018-2023). Nueva York, NY: Curran Associates.

Anglin, J. M. (1993). Vocabulary development: a morphological analysis. Monographs of the Society for Research in Child Development, 58, 1-186. https://doi.org/10.2307/1166112

Auza, A. (2005). Morfología nominal en el niño de 3:7 a 4:0 años: ¿cuál estrategia se emplea en la adquisición de una clase léxica? Lingüística Mexicana, 2(1), 105-114.

Auza, A. (2006). La transparencia morfológica y conceptual en la adquisición de términos centrales y periféricos. Lenguaje, 34, 71-95. https://doi.org/10.25100/lenguaje.v34io.4842

Bangs, K. E. y Binder, K. S. (2016). Morphological awareness intervention: improving spelling, vocabulary, and reading comprehension for adult learners. Journal of Research and Practice for Adult Literacy, Secondary, and Basic Education, 5(1), 49-56.

Baralo, M. (2007). Adquisición de palabras: redes semánticas y léxicas. En C. Pastor (Coord.), Actas del Programa de formación para profesorado de español como lengua extranjera 2006-2007 (pp. 384-399). Múnich: Instituto Cervantes.

Barriga, R. (2002). Estudios sobre habla infantil en los años escolares: un solecito calientote. Ciudad de México: El Colegio de México. https://doi.org/10.2307/j.ctv47w9cc

Baumann, J. F. (2008). Vocabulary and reading comprehension: the nexus of meaning. En S.E. Israel, y G.G. Duffy (Eds.), Handbook of research on reading comprehension (pp. 323-34). Nueva York, NY: Routledge.

Baumann J. F., Ware, D. y Edwards, E. C. (2007). 'Bumping into spicy, tasty words that catch your tongue': a formative experiment in vocabulary instruction. The Reading Teacher, 61(2), 108122. https://doi.org/10.1598/rt.61.2.1 
Beck, I.L., McKeown, M. G. y Kucan, L. (2008). Creating robust vocabulary. Nueva York: The Guilford Press.

Bowers, P. N., Kirby, J. R. y Deacon, S. H. (2010). The effects of morphological instruction on literacy skills: a systematic review of the literature. Review of Educational Research, 8O(2), 144-1 79. https://doi.org/10.3102/0034654309359353

Carlisle, J. F. (2004). Morphological processes that influence learning to read. En C. A. Stone, E. R. Silliman, B. J. Ehren, y K. Apel (Eds.), Handbook of language y literacy (pp. 318-339). Nueva York, NY: The Guilford Press.

Carmiol, A. M., Ríos, M. y Sparks, A. (2013). La relación entre habilidades prelectoras y habilidades narrativas en niños y niñas preescolares costarricenses: aportes para un enfoque comprensivo de la alfabetización emergente. En A. Auza, y K. Hess (Eds.), ¿Qué me cuentas? Narraciones y desarrollo lingüistico en niños hispanohablantes (pp. 318-339). Ciudad de México: DeLaurel-Universidad Autónoma de Querétaro.

Cassany, D., Luna, M. y Sanz, G. (1994). Enseñar lengua. Barcelona: Graó.

Clark, E. V. (2009). First language acquisition. Cambridge, MA: Cambridge University Press. https://doi.org/10.1017/cbo9780511806698

Clark, E. V. (2014). Two pragmatic principles in language use and acquisition. En D. Matthews (Ed.), Pragmatic development in first language acquisition (pp. 105-120). Amsterdam: John Benjamins. https://doi.org/10.1075/tilar.10.07cla

Corrêa, L. y Peruzzi, M. M. (2018). Há uma relação específica entre consciência morfológica e reconhecimento de palavras? Psico USF, Bragança Paulista, 18(2), 241-248. https://doi.org/10.1590/s1413-82712013000200008

Crystal, D. (1987). Teaching vocabulary: the case for a semantic curriculum. Child Language Teaching and Therapy, 3(1), 40-56. https://doi.org/10.1177/026565908700300103

De Freitas, P. V., Mota, M. M. y Deacon, S. H. (2018). Morphological awareness, word reading, and reading comprehension in Portuguese. Applied Psycholinguistics, 39(3), 507-525. https://doi.org/10.1017/s0142716417000479

Dockrell, J. E. y Messer, D. (2004). Lexical acquisition in the early school years. En R.A. Berman (Ed.), Language development across childhood and adolescence (pp. 35-52). Amsterdam: John Benjamins. https://doi.org/10.1075/tilar.3.06doc

Gómez, V., Sotomayor, C., Bedwell, P., Domínguez, A. M. y Jéldrez, E. (2016). Analysis of lexical quality and its relation to writing quality for 4th grade, primary school students in Chile. Reading and Writing, 29, 1317-1336. https://doi.org/10.1007/s1 1145-016-9637-9

González, R. O. (2014). Habilidades lingüísticas de los estudiantes de primer ingreso a las instituciones de educación superior. Ciudad de México: ANUIES.

Goodwin, A.P., Petscher, Y., Carlisle, J. F. y Mitchell, A. M. (2017). Exploring the dimensionality of morphological knowledge for adolescent readers. Journal of Research in Reading, $40(1)$, 91-117. https://doi.org/10.1111/1467-9817.12064

Graves, M.F. (2006). The vocabulary book. Nueva York, NY: Teachers College Press.

Gutiérrez, R. y Díez, A. (2018). Conciencia fonológica y desarrollo evolutivo de la escritura en las primeras edades. Educación XXI, 21(1), 395-416. https://doi.org/10.5944/educxx 1.20212

Hess, K. (2013). Desarrollo léxico en la adolescencia: un análisis de sustantivos en narraciones orales y escritas. Actualidades en Psicología, 27(115), 113-127.

https://doi.org/10.15517/ap.v27i115.8316 
Hess, K. (2018). Desarrollo de la capacidad para reflexionar sobre la morfología de las palabras en los años escolares. En C. Rojas \& V. Oropeza (Eds.). Diferencias individuales en la adquisición del lenguaje. Factores lingüísticos, cognitivos, socioambientales (pp. 253-293). Ciudad de México: IIF-UNAM.

Hoff, E. (2014) Language development. Belmont: Wadsworth.

Jaichenco, V. y Wilson, M. (2013). El rol de la morfología en el proceso de aprendizaje de la lectura en español. Interdisciplinaria, 30(1), 85-99. https://doi.org/10.16888/interd.2013.30.1.5

Jisa, H. (2004). Growing into academic French. En R. A. Berman (Ed.), Language development across childhood and adolescence (pp. 135-161). Amsterdam: John Benjamins. https://doi.org/10.1075/tilar.3.10jis

Kelley, J. G., Lesaux, N. K., Kieffer, M. J. y Faller, S. E. (2010). Effective academic vocabulary instruction in the urban middle school. The Reading Teacher, 64(1), 5-14. https://doi.org/10.1598/rt.64.1.1

Kieffer, M. J. y Lesaux, N. K. (2007). Breaking down words to build meaning: morphology, vocabulary, and reading comprehension in the urban classroom. The Reading Teacher, 61(2), 134-144. https://doi.org/10.1598/rt.61.2.3

Kieffer, M. J. y Lesaux, N. K. (2012). Effects of academic language instruction on relational and syntactic aspects of morphological awareness for sixth graders from linguistically diverse backgrounds. The Elementary School Journal, 112(3), 519-545. https://doi.org/10.1086/663299

Larsen, J. A. y Nippold, M. A. (2007). Morphological analysis in school-age children: dynamic assessment of a word learning strategy. Language, Speech, and Hearing Services in Schools, 38, 201-212. https://doi.org/10.1044/0161-1461(2007/021)

Levie, R., Ben-Zvi, G. y Ravid, D. (2016). Morpho-lexical development in language impaired and typically developing Hebrew-speaking children from two SES backgrounds. Reading and Writing, 3O(5), 1035-1064. https://doi.org/10.1007/s11145-016-9711-3

Li, L. y Wu, X. (2015). Effects of metalinguistic awareness on reading comprehension and the mediator role of reading fluency from Grades 2 to 4. PloS ONE, 1O(3), 1-16. https://doi.org/10.1371/journal.pone.0114417

Llauradó, A. y Tolchinsky, L. (2013). Growth of text-embedded lexicon in Catalan: from childhood to adolescence. First Language, 33(6), 628-653. https://doi.org/10.1177/0142723713508861

Mahony, D. Singson, M. y Mann, V. (2000). Reading ability and sensitivity to morphological relations. Reading and Writing: An Interdisciplinary Journal, 12, 191-218.

Mancilla-Martinez, J. y Lesaux, N. K. (2011). Early home language use and later vocabulary development. Journal of Educational Psychology, 103(3), 535-546. https://doi.org/10.1037/a0023655

Marchant, T., Lucchini, G., Cuadrado, B. y Fundación Educacional Arauco (2007). ¿Por qué leer bien es importante? Asociación del dominio lector con otros aprendizajes. Psykhe, 16(2), 316. https://doi.org/10.4067/s07 18-22282007000200001

Martín, R. A. (2018). Modelos de aprendizaje léxico basados en la morfología derivativa. RILCE, 34(1), 262-285. https://doi.org/10.15581/008.34.1.262-85

Murphy, A., Franklin, S., Breen, A., Hanlon, M., McNamara, A., Bogue, A. y James, E. (2017). A whole class teaching approach to improve the vocabulary skills of adolescents attending 
mainstream secondary school in areas of socioeconomic disadvantage. Child Language Teaching and Therapy, 33(2), 129-144. https://doi.org/10.1177/0265659016656906

Nagy, W. y Townsend, D. (2012). Words as tools: learning academic vocabulary as language acquisition. Reading Research Quarterly, 47(1), 91-108. https://doi.org/10.1002/rrq.011

Nippold, M. A. (2007). Later language development: school-age children, adolescents, and young adults. Austin: Pro-Ed. https://doi.org/10.1016/b0-08-044854-2/00852-x

Nippold, M. A. y Sun, L. (2008). Knowledge of morphologically complex words: A developmental study of older children and young adolescents. Language, Speech, and Hearing Services in Schools, 39, 365-373. https://doi.org/10.1044/0161-1461(2008/034)

Pacheco, M. B. y Goodwin, A. P. (2013). Putting two and two together: middle school students' morphological problem-solving strategies for unknown words. Journal of Adolescent y Adult Literacy, 56(7), 541-553. https://doi.org/10.1002/jaal.181

Pence-Turnbull, K. L. y Justice, L. M. (2008). Language development from theory to practice. Upper Saddle River, NJ: Pearson-Prentice Hall.

Ravid, D. (2004). Derivational morphology revisited: later lexical development in Hebrew. En R.A. Berman (Ed.), Language development across childhood and adolescence (pp. 53-81). Amsterdam: John Benjamins. https://doi.org/10.1075/tilar.3.07rav

Ravid, D. y Geiger, V. (2009). Promoting morphological awareness in Hebrew-speaking gradeschoolers: an intervention study using linguistic humor. First Language, 29(1), 81-112. https://doi.org/10.1177/0142723708097483

Rojas, M. (2005). Una responsabilidad escolar olvidada: El desarrollo del componente léxico. Revista Educación, 29(1), 31-44. https://doi.org/10.15517/revedu.v29i1.2021

Rojas, M. (2006). Esbozo de lineamientos conceptuales para la enseñanza y aprendizaje del léxico. Revista Educación, 30(2), 9-29. https://doi.org/10.15517/revedu.v30i2.2227

Schleppegrell, M. J. (2004). The language of schooling: a functional linguistics perspective. Mahwah, NJ: Lawrence Erlbaum. https://doi.org/10.4324/9781410610317

Snow, C. E. y Uccelli, P. (2009). The challenge of academic language. En D.R. Olson, y N. Torrance (Eds.), The Cambridge handbook of literacy (pp. 112-133). Cambridge, MA: Cambridge University Press. https://doi.org/10.1017/cbo9780511609664.008

Sparks, E. y Deacon, S.H. (2015). Morphological awareness and morphological acquisition: a longitudinal examination of their relationship in English-speaking children. Applied Psycholinguistics, 36, 299-321. https://doi.org/10.1017/s0142716413000246

St. John, P. y Vance, M. (2014). Evaluation of a principled approach to vocabulary learning in mainstream clases. Child Language Teaching and Therapy, 30(3), 255-271. https://doi.org/10.1177/0265659013516474

Stahl, S. A. (1999). Vocabulary development. Brookline, MA: Brookline Books.

Stahl, S. A. y Shiel T. G. (1992). Teaching meaning vocabulary: productive approaches for poor readers. Reading and Writing Quarterly, 8, 223-241. https://doi.org/10.1080/0748763920080206

Thorne, C., Morla, K., Uccelli, P., Nakano, T., Mauchi, B., Landeo, L. et al. (2013). Efecto de una plataforma virtual en comprensión de lectura y vocabulario: una alternativa para mejorar las capacidades lectoras en primaria. Revista de Psicología, 31(1), 3-35. 
Tighe, E. L. y Binder, K. S. (2015). An investigation of morphological awareness and processing in adults with low literacy. Applied Psycholinguistics, 36, 245-273.

https://doi.org/10.1017/sO142716413000222

Zwiers, J. (2008). Building academic language: essential practices for content classrooms, San Francisco, CA: Jossey Bass.

\section{Anexo I. Instrumento que se presentó a los participantes de la investigación durante el pre-test y el pos-test (Forma A)}

Nombre:

\begin{tabular}{|c|c|c|}
\hline Palabra & Significado de las partes & Significado de la palabra completa \\
\hline SUBSUELO & $\begin{array}{l}\text { SUB - debajo de } \\
\text { SUELO - piso }\end{array}$ & lugar debajo del piso \\
\hline PREOPERATORIO & $\begin{array}{l}\text { PRE: antes de } \\
\text { OPERAT: que ejecuta algo, que opera } \\
\text { algo } \\
\text { ORIO: característica de }\end{array}$ & $\begin{array}{l}\text { característica de hacerse antes de una } \\
\text { operación }\end{array}$ \\
\hline \multicolumn{3}{|l|}{ HIDRÓGENO } \\
\hline \multicolumn{3}{|l|}{ DERRUMBE } \\
\hline \multicolumn{3}{|l|}{ CARTOGRAFÍA } \\
\hline \multicolumn{3}{|l|}{ REFORMA } \\
\hline \multicolumn{3}{|l|}{ ENDOMETRIO } \\
\hline \multicolumn{3}{|l|}{ TOPOGRAFÍA } \\
\hline \multicolumn{3}{|l|}{ FERMENTACIÓN } \\
\hline \multicolumn{3}{|l|}{ BIÓXIDO } \\
\hline \multicolumn{3}{|l|}{ ARIDOAMÉRICA } \\
\hline \multicolumn{3}{|l|}{ RECICLAJE } \\
\hline \multicolumn{3}{|l|}{ IMPUESTO } \\
\hline \multicolumn{3}{|l|}{ POBLAMIENTO } \\
\hline \multicolumn{3}{|l|}{ ANTROPOLOGÍA } \\
\hline \multicolumn{3}{|l|}{ MANDATARIO } \\
\hline \multicolumn{3}{|l|}{ METANO } \\
\hline \multicolumn{3}{|l|}{ PROCARIOTE } \\
\hline \multicolumn{3}{|l|}{ EBULLICIÓN } \\
\hline \multicolumn{3}{|l|}{ INTERGLACIAL } \\
\hline \multicolumn{3}{|l|}{ RECONQUISTA } \\
\hline CIVILIZACIÓN & & \\
\hline
\end{tabular}




\begin{tabular}{|l|l|l|}
\hline AERONÁUTICA & & \\
\hline GLACIACIÓN & & \\
\hline HIDROCARBURO & & \\
\hline BIÓSFERA & & \\
\hline MESOZÓICA & & \\
\hline FEDERALISMO & & \\
\hline INTOXICACIÓN & & \\
\hline
\end{tabular}

\section{Anexo II. Leyendas de Figuras}

- Figura 1. Ejemplo de algunas de las palabras inventadas por una pareja de estudiantes durante la elaboración del Diccionario de Palabras Inventadas

- Figura 2. Cantidad total de respuestas por tipo para la segmentación de palabras en el grupo experimental. El eje de la "x" muestra el tipo de respuesta ( $\mathrm{A}=$ segmentación correcta, $\mathrm{B}=$ segmentación incorrecta pero recupera al menos un morfema correctamente, $\mathrm{C}=$ segmentación asistemática, silábica o no segmenta) y el eje de la "y" indica el número de respuestas. El total de respuestas posibles era 1026.

- Figura 3. Cantidad total de respuestas por tipo para la segmentación de palabras en el grupo control. El eje de la "x" muestra el tipo de respuesta (A=segmentación correcta, $\mathrm{B}=$ segmentación incorrecta pero recupera al menos un morfema correctamente, $\mathrm{C}=$ segmentación asistemática, silábica o no segmenta) y el eje de la "y" indica el número de respuestas. El total de respuestas posibles era 1026.

- Figura 4. Cantidad total de respuestas por tipo para la obtención del significado de palabras en el grupo experimental. El eje de la " $x$ " muestra el tipo de respuesta ( $\mathrm{X}=$ recupera el significado de la palabra y los morfemas, $\mathrm{Y}=$ recupera el significado de la palabra o de algún morfema, $Z=$ no recupera el significado de la palabra ni de ningún morfema) y el eje de la "y" indica el número de respuestas. El total de respuestas posibles era 1026.

- Figura 5. Cantidad total de respuestas por tipo para la obtención del significado de palabras en el grupo control. El eje de la "x" muestra el tipo de respuesta ( $\mathrm{X}=$ recupera el significado de la palabra y los morfemas, Y=recupera el significado de la palabra o de algún morfema, $Z=$ no recupera el significado de la palabra ni de ningún morfema) y el eje de la "y" indica el número de respuestas. El total de respuestas posibles era 1026. 


\section{Breve $\mathrm{Cv}$ de la autora}

\section{Karina Hess Zimmermann}

Licenciada en Educación Especial por la Universidad de las Américas, A.C. y doctora en Lingüística por El Colegio de México. Actualmente es profesora-investigadora de la Facultad de Psicología de la Universidad Autónoma de Querétaro y pertenece al grupo de docentes de la Maestría en Aprendizaje de la Lengua y las Matemáticas, un programa de formación para profesores de Educación Básica. Sus líneas de investigación giran en torno al desarrollo lingüístico en los años escolares y a la manera en que la escuela puede incidir en el desarrollo del lenguaje de los estudiantes. ORCID ID: 0000-0002-8960-2205. Email: karinahess@hotmail.com. 